\title{
Diet and longevity in the balance
}

Thomas Flatt

\section{Dietary restriction promotes longevity but impairs fecundity in many organisms. When the amino acids in a diet are fine-tuned, however, lifespan can be increased without loss of fecundity - at least in fruitflies.}

It's common wisdom that eating the right food, and not too much of it, is good for you. Dietary restriction - reduced food intake without malnutrition - indeed prolongs lifespan in organisms ranging from yeast, worms and flies to rodents, monkeys and possibly humans. But dietary restriction also has its costs: it often impairs fecundity, possibly because maintenance of the soma (the non-germline parts of an organism), and thus long life, are incompatible with the metabolic demands of reproduction in such circumstances. Biologists have long thought that an organism's response to food shortage is an evolutionary device that allows individuals to survive a famine by diverting resources away from reproduction and reallocating them to essential functions for survival ${ }^{1,2}$.

On page 1061 of this issue, Grandison et al. ${ }^{3}$ report that this idea is almost certainly wrong. They find that dietary amino acids are responsible for shortening lifespan and increasing reproduction in the fruitfly Drosophila melanogaster, but that both longevity and fecundity can be maximized when intake of these nutrients is finely tuned.

Great strides have recently been made in our understanding of dietary and caloric restriction $^{4-8}$. It has become clear that rich diets shorten life, not because of excess calories but rather because of dietary imbalance, with lifespan and fecundity being maximized at different nutritional optima ${ }^{5}$. Specific nutrients are implicated in dietary restriction, especially amino acids, the building blocks of proteins. For example, reducing the intake of casein, a major amino-acid source, extends lifespan but decreases fecundity in Drosophila ${ }^{6}$. Similarly, methionine restriction promotes longevity in flies, rats and mice ${ }^{7,8}$. Although these studies suggest that amino-acid deficiency might account for the effects of dietary restriction, Grandison et al. ${ }^{3}$ have gone several steps further.

The authors have identified the specific nutrients that modulate lifespan and reproduction by manipulating all of the components of a defined diet. In a series of painstaking experiments, they fed female flies a restricted diet that extends lifespan at the expense of

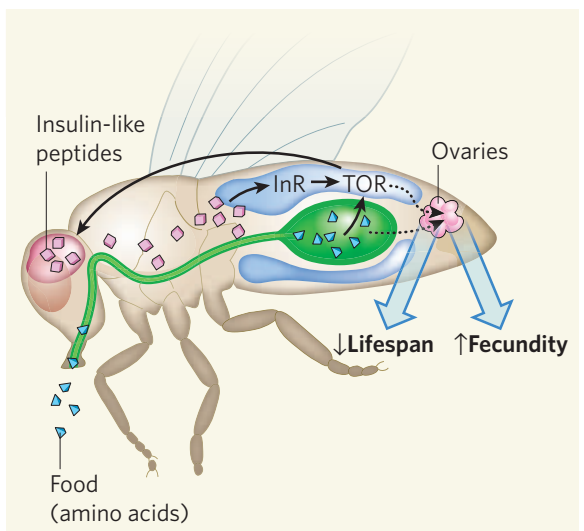

Figure 1 | Signalling pathways possibly influenced by dietary amino acids in Drosophila. Grandison et al. ${ }^{3}$ show that the lifespan-increasing and fecundity-reducing effects of dietary restriction can be attributed to amino-acid limitation. During full feeding, amino acids tend to shorten lifespan and promote egg production. These effects seem to operate via insulin-like signalling, mediated by insulin-like peptides produced in the brain, as mutant flies lacking insulin receptors are protected against the lifespan shortening and loss of fecundity that usually accompany full feeding. During development and growth, insulin signalling interacts with the nutrient-sensing TOR pathway. Because TOR signalling has been implicated in dietary restriction ${ }^{4,9}$, insulin/TOR crosstalk could integrate the effects of dietary amino acids on lifespan and reproduction. Blue, fat body; green, digestive system; InR, insulin-like receptor; TOR, target of rapamycin.

fecundity, and then tried to restore the shortlife and high-fecundity characteristics of fully fed flies by adding back specific nutrients. Adding carbohydrates, lipids or vitamins made no difference. But adding amino acids shortened lifespan and increased egg production to the level observed under full feeding. The question then was whether all amino acids were contributing equally to this astonishing effect.

Amino acids can be divided into two types: non-essential (those that can be synthesized by an organism) and essential (those that must be supplied in the diet). Grandison et al. ${ }^{3}$ found that adding all non-essential amino acids only marginally shortened lifespan and did not change fecundity, whereas adding all essential amino acids decreased lifespan and increased egg production as much as combining all amino acids or full feeding. The authors next manipulated each essential amino acid individually. Remarkably, methionine alone increased fecundity as much as full feeding but without reducing lifespan. In fact, no single amino acid shortened lifespan to the fully fed state. Yet, adding all essential amino acids except methionine failed to reduce lifespan, suggesting that methionine together with one or several other essential amino acids is responsible for the lifespan-shortening effect of full feeding. This finding is consistent with work showing that methionine restriction promotes fly longevity ${ }^{7}$.

So how does methionine, either by itself or in combination with other amino acids, influence lifespan ${ }^{7,8}$ ? One possibility is that methionine restriction counters oxidative damage: dietary methionine deficiency increases the levels of the antioxidant glutathione in rats and protects mice from oxidative liver-cell injury ${ }^{8}$. Another explanation might be that methionine restriction extends lifespan by reducing signalling through a major regulator of longevity in worms, flies and rodents ${ }^{2,4,7,8}$, the IIS - insulin/ insulin-like growth factor (IGF) - pathway. Indeed, methionine-deficient mice show lowered levels of IGF-1 and insulin ${ }^{8}$. This is particularly interesting in the light of Grandison and colleagues' results: adding back essential amino acids to the diet decreased lifespan strongly in normal flies, but only slightly in mutant flies lacking the insulin-like receptor that mediates IIS activity. Addition of methionine did not promote fecundity in these mutants. These results imply that the IIS pathway mediates key effects of amino acids on ageing and reproduction (Fig. 1).

During development and growth, IIS is known to interact with 'target of rapamycin' (TOR) signalling, a nutrient-sensing pathway also implicated in dietary restriction ${ }^{4,9}$, and it is tempting to speculate that amino acids act through TOR to affect lifespan (Fig. 1). In line with this idea, a repressor protein, $4 \mathrm{E}-\mathrm{BP}$, 
which lies downstream of TOR, is required for lifespan extension in flies when their diet is restricted ${ }^{9}$. Furthermore, dietary amino acids stimulate insulin secretion in the brain through a TOR-dependent mechanism in the Drosophila fat body, the equivalent of mammalian liver and adipose tissue ${ }^{10}$. The connections between dietary amino acids, IIS and TOR clearly merit further investigation.

The findings of Grandison et al. ${ }^{3}$ also have implications for understanding the trade-off between survival and reproduction. Although reproduction shortens lifespan in various organisms ${ }^{2,11}$, the mechanisms involved have remained elusive. The most widely accepted view is that when resources are invested in reproduction, they are unavailable for somatic maintenance and survival ${ }^{1}$. Alternatively, reproduction might inflict direct somatic damage independent of nutrient allocation ${ }^{11}$. Both explanations, however, seem to be at odds with the observations of Grandison et al. because methionine alone can restore full fecundity to long-lived flies on an otherwise restricted diet. If the diet is correctly balanced, both fecundity and lifespan can be maximized, without any apparent trade-off between them.

But are things really this simple? Probably not. Although dietary restriction can extend lifespan when flies or worms are sterilized ${ }^{2}$, it does not further improve the lifespan of long-lived worms lacking germ cells ${ }^{12}$. Dietary restriction and reproduction might thus converge on the same mechanisms regulating lifespan ${ }^{12}$. That we don't yet fully understand the intricate connections between diet, metabolism, reproduction and lifespan is underscored by the finding that ablation of germline cells extends lifespan by decreasing fat storage in the worm Caenorhabditis elegans ${ }^{13}$. Nonetheless, if the present results ${ }^{3}$ in the fly are generally applicable, even mammals might be able to enjoy a long life without loss of fecundity by virtue of a suitably balanced diet.

Thomas Flatt is in the Department of

Biomedical Sciences, Institute of Population

Genetics, University of Veterinary

Medicine Vienna, Veterinärplatz 1, A-1210 Vienna, Austria.

e-mail: thomas.flatt@vetmeduni.ac.at

1. Kirkwood, T. B. L. Nature 270, 301-304 (1977)

2. Flatt, T. \& Schmidt, P. Biochim. Biophys. Acta 1790, 951-962 (2009).

3. Grandison, R. C., Piper, M. D. W. \& Partridge, L. Nature 462, 1061-1064 (2009).

4. Mair, W. \& Dillin, A. Annu. Rev. Biochem. 77, 727-754 (2008).

5. Skorupa, D. A., Dervisefendic, A., Zwiener, J. \& Pletcher, S. D. Aging Cell 7, 478-490 (2008).

6. Min, K.-J. \& Tatar, M. Mech. Ageing Dev. 127, 643-646 (2006).

7. Troen, A. M. et al. Age 29, 29-39 (2007).

8. Miller, R. A. et al. Aging Cell 4, 119-125 (2005).

9. Zid, B. M. et al. Cell 139, 149-160 (2009).

10. Géminard, C., Rulifson, E. J. \& Léopold, P. Cell Metab. 10, 199-207 (2009).

11. Tatar, M. \& Carey, J. R. Ecology 76, 2066-2073 (1995).

12. Crawford, D., Libina, N. \& Kenyon, C. Aging Cell 6, 715-721 (2007).

13. Wang, M. C., O'Rourke, E. J. \& Ruvkun, G. Science 322, 957-960 (2008). 